University of Nebraska - Lincoln

DigitalCommons@University of Nebraska - Lincoln

Faculty Publications from the Department of Engineering Mechanics

Mechanical \& Materials Engineering,

Department of

2009

\title{
Evaporation induced two-dimensional buckling within liquid droplet
}

Ziguang Chen

University of Nebraska-Lincoln, chen_ziguang@163.com

Maozi Liu

Agilent Technologies Inc., Santa Clara, CA

Gang-yu Liu

University of California, Davis

Li Tan

University of Nebraska-Lincoln, Itan4@unl.edu

Follow this and additional works at: https://digitalcommons.unl.edu/engineeringmechanicsfacpub

Part of the Mechanical Engineering Commons

Chen, Ziguang; Liu, Maozi; Liu, Gang-yu; and Tan, Li, "Evaporation induced two-dimensional buckling within liquid droplet" (2009). Faculty Publications from the Department of Engineering Mechanics. 73. https://digitalcommons.unl.edu/engineeringmechanicsfacpub/73

This Article is brought to you for free and open access by the Mechanical \& Materials Engineering, Department of at DigitalCommons@University of Nebraska - Lincoln. It has been accepted for inclusion in Faculty Publications from the Department of Engineering Mechanics by an authorized administrator of DigitalCommons@University of Nebraska - Lincoln. 


\title{
Evaporation induced two-dimensional buckling within liquid droplet
}

\author{
Ziguang Chen, ${ }^{1}$ Maozi Liu, ${ }^{2}$ Gang-yu Liu, ${ }^{3}$ and Li $\operatorname{Tan}^{1, a)}$ \\ ${ }^{1}$ Department of Engineering Mechanics, Nebraska Center for Materials and Nanoscience, \\ University of Nebraska, Lincoln, Nebraska 68588, USA \\ ${ }^{2}$ Agilent Technologies Inc., 5301 Stevens Creek Blvd., Santa Clara, California 95051, USA \\ ${ }^{3}$ Department of Chemistry, University of California, One Shields Avenue, Davis, California 95616, USA
}

(Received 9 September 2009; accepted 3 November 2009; published online 2 December 2009)

\begin{abstract}
Evaporation of a liquid droplet containing a thin layer of surfactants atop could generate two-dimensional buckling on surfaces. Herringbone features were produced via embedded nanoparticle beds. We assign transient surface tension as the driving force for such a phenomenon. Considering the surfactant layer as a thin elastic film, a continuum model is employed to calculate the mechanical properties of the layer. Particularly, we estimated an elastic modulus of $4 \mathrm{GPa}$ for the surfactant layer, indicating rather strong mechanical properties of these small molecules when they are close packed to form supramolecules via noncovalent binding. () 2009 American Institute of Physics. [doi:10.1063/1.3269930]
\end{abstract}

Buckling or wrinkling exists in numerous forms over wide length scales and their common forms include wrinkling of human skin, shrinking surfaces of dried fruits, and even the formation of mountain ridges. This phenomenon has been utilized in the field of nanofabrication, where buckling of a stiff thin film on a compliant substrate might be useful in optoelectronics, generating applications from nature-mimic compound eyes ${ }^{1}$ to stretchable metal oxide semiconductors. ${ }^{2}$ Generally, all these bucklings occurred in solid thin films supported by solid substrates. We learned that, beyond a critical strain, the elastic energy required to compress the solid film can be reduced by an out-of-plane bending, or the formation of buckling. Minimization of the total elastic energy leads to scaling relationships between the amplitude and wavelength of those buckled features, ${ }^{3,4}$ which can be even applied in mechanical characterizations of thin polymer films. ${ }^{5,6}$ In contrast, buckling of a thin film on liquid surfaces is rarely studied. ${ }^{7}$

In this letter, we investigate the formation of twodimensional (2D) buckling regulated via surfactant monolayers. Surfactants are amphiphlic molecules that have been widely used in organic/inorganic hybrid systems during nanofabrication. When concentrated molecules are dispersed in an aqueous liquid, a monolayer could cover the entire air and water interface, stabilizing the liquid droplet by reducing the surface tension. Presumably, when the liquid volume is condensed, a compression could be applied to the monolayer, inducing periodic topography features or buckling. The periodic buckling was discovered in the evaporation of a water droplet containing a very small amount $(1.0 \mathrm{wt} \%)$ of monodispersed polystyrene spheres (Duke Scientific, Inc.). The mean sphere size is 30 or $80 \mathrm{~nm}$ with a standard deviation of $18 \%$. Among this droplet, $0.1 \mathrm{wt} \%$ of sodium dodecylsulfate was also added as surfactants to stabilize the nanoparticles before evaporation. And we record the entire evaporation process under an optical microscope (ML8000 Meiji) equipped with a digital camera (Moticam 2000).

\footnotetext{
${ }^{\text {a) }}$ Author to whom correspondence should be addressed. Electronic mail: 1tan4@unl.edu. FAX: 402-472-8292. Tel.: 402-472-4018.
}

It is well known that, during the evaporation of a pure water droplet, the contact angle gradually increases as the droplet shrank. In contrast, the contact angle change in our droplet (weight $\approx 30 \mathrm{mg}$ ) differs due to the presence of a mixture of nanoparticles within and a monolayer of surfactants at the air-liquid interface. The droplet initially spread out as a circle with a diameter of $1.0 \mathrm{~cm}$ and a contact angle $(\alpha)$ of $15^{\circ}$ [phase 1, Fig. 1(a)]. Next, nanoparticles were driving from the inside of the droplet and piled as aggregated precipitates at the inner rim of the droplet [phase 2, Fig. 1(a)]. This essentially delivers a water reservoir surrounded by a bank of nanoparticles and a further evaporation created the so called "coffee-ring," 8 where both the radius of the droplet $R$ and the contact angle $\alpha$ of the droplet remain relatively constant. In both phases, the liquid edge is always standing statically on the substrate (glass). Overall force acting at this edge along the horizontal direction is, $\Sigma F_{x}$ $=f_{L V} \cos \alpha+f_{S L}-f_{V S}=0$, where $f_{L V}, f_{V S}$, and $f_{S L}$ are the ten-

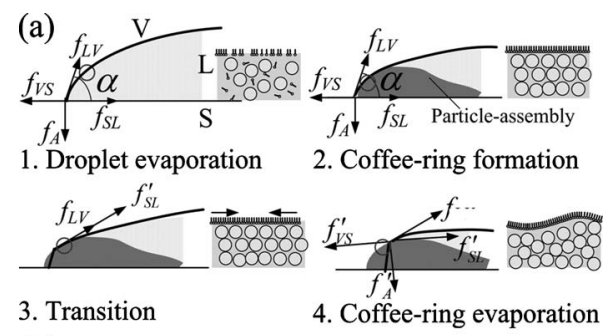

(b)

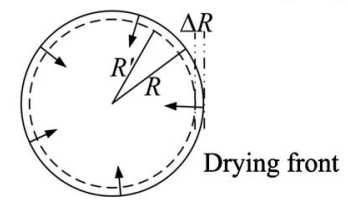

FIG. 1. (a) Schematic illustration of the 2D buckling formation process and the corresponding free-body diagrams. Phase 1, evaporation stage $\left(\Sigma F_{x}\right.$ $\left.=f_{L V} \cos \alpha+f_{S L}-f_{V S}=0\right)$; phase 2, coffee-ring formation $\left(\Sigma F_{x}=f_{L V} \cos \alpha\right.$ $\left.+f_{S L}-f_{V S}=0\right)$; phase 3 , transition stage $\left(\Sigma F_{x} \sim f_{L V}+f_{S L}^{\prime}>0\right)$; and phase 4 , coffee-ring evaporation $\left(\sum F_{x} \sim f_{L V} \cos \alpha^{\prime}+f_{S L}^{\prime}-f_{V S}^{\prime}=0\right)$. A compressive transient surface force is experienced atop the surfactant layer in phase 3 , leading to the $2 \mathrm{D}$ buckling. (b) A top view on liquid front moving toward the center during the transition stage. Shaded areas with a dark color represent aggregated nanoparticles at the corner of the liquid droplet, while areas with a light gray color for water. 


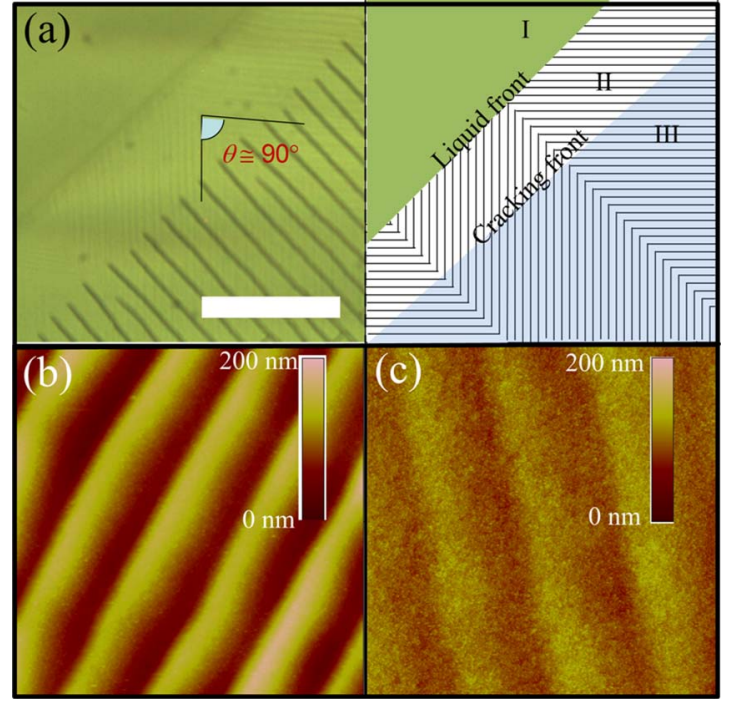

FIG. 2. (Color online) (a) An optical microscopic image (left) and its illustration (right) of a drying suspension with nanoparticles. An array of herringbonetype buckling feature formed prior to cracking. Scale bar: $100 \mu \mathrm{m}$. [(b) and (c)] AFM images $\left(20 \times 20 \mu \mathrm{m}^{2}\right)$ of the buckled feature near the droplet edge. The diameter of the nanoparticle is $30 \mathrm{~nm}$ in (b) and $80 \mathrm{~nm}$ in (c).

sion forces among interfaces of liquid-vapor, vapor-substrate, and substrate-liquid, respectively. The vertical component of $f_{L V}$ is neglected since it can be canceled by an adhesive force $f_{A}$ from the substrate. Before transition to phase 2, the particles are dispersed inside the water droplet and the surfactant has not fully covered the water surface yet. Gradually, the surfactants on the water surface increased with the continued removal of water, and at the end of this phase, a monolayer of surfactants finally covered the entire air/water interface with a much reduced surface area. After the surface on the water reservoir becomes flat in phase 2, further evaporation will lift the drying front from the glass substrate to the top surface of the particle bank. This delivered a significant transition phase as shown in phase 3, Fig. 1(a), where the static equilibrium is broken to result a nonzero force, or $f_{L V}+f_{S L}^{\prime}$. As a consequence, the surface of surfactant monolayers will be compressed, introducing buckling to the monolayer and forcing a deformation to the nanoparticles underneath. Essentially, what we observed on nanoparticles is a direct result of buckling from surfactant film. And this feature manifests itself after the removal of water. After this transition phase, the evaporation front reaches its new equilibrium state as shown in phase 4, Fig. 1(a). Figure 1(b) shows a top view for this transition, where $R$ is the diameter of the droplet at the end of phase 2 and $R^{\prime}$ is the diameter at the beginning of phase 4 .

Three regions with clear optical contrasts were spotted atop the particle bank as shown in Fig. 2(a), namely, region I as the liquid reservoir, region II as the evaporation front and region III as dried film with cracks. The buckling pattern in evaporation front (II) was rarely reported ${ }^{9}$ and is the focus of our following discussion. The drying process associated with cracking, on the other hand, has been discussed by others. ${ }^{10}$ Here we mainly discuss the evaporation front, where buckling pattern forms before the cracking in region III [see Fig. 2(a)]. First thing we noticed in region II is the zig-zag patterns with periodically protruding ridges echoing the phenomenon of buckling. Second, these features smoothly ex- tended into the liquid reservoir from region I to III, indicating the buckling occurred long before the formation of those three regions. We used tapping mode of an atomic force microscope (AFM) to characterize region II, as shown in Figs. 2(b) and 2(c). In both panels, nanospheres of 30 and $80 \mathrm{~nm}$, respectively, demonstrated waving or wrinkling features over an area approximately $20 \times 20 \mu \mathrm{m}^{2}$. On $30 \mathrm{~nm}$ particle film, the wavelength is $4.6 \mu \mathrm{m}$, and the amplitude is $50 \mathrm{~nm}$, while on $80 \mathrm{~nm}$ particle film, they are, respectively, $5.5 \mu \mathrm{m}$ and $25 \mathrm{~nm}$. All these wavelengths match with a prediction by Milner et al. ${ }^{11}$ that Langmuir monolayers may buckle like a plate or beam.

One might suggest that water wave or layered sliding among packed nanoparticles could have contributed to the observed 2D features, our verdict is otherwise. In particular, water wave usually bears a sinusoidal pattern, radially distributed from the center of the droplet toward the outer edge. Accordingly, concentric rings, instead of the observed zigzag feature, should be revealed on surfaces of the particle bank. Layered sliding, on the other hand, should generate terraces of close-packed particles with a sharp step edge. These two features are all clearly different from a zig-zag feature. Hence, both motions are excluded from the cause of buckling, and the surfactant is the only component that could play a dominant role in the formation of these $2 \mathrm{D}$ features. Since the buckling requires the stiffness of film far larger than that of underlying substrate, the observed buckling implies that the nanoparticles formed a noncontact network rather than contacted ones. Seemingly surprising, recent observations in a drying film supported this argument. ${ }^{12}$

The buckling features are best modeled using a quasiquantitative means. The dynamic surface area change can be shown in Fig. 1(b). Such a change caused a reduction of the surface free energy of the liquid film, which can be described as $\Delta G=\gamma \Delta A$, where $\gamma$ is the surface tension of the film and $\Delta A=2 \pi R \Delta R$ is the area change due to the evaporation. The axial and tangential compressive strain are, respectively, $\varepsilon_{1}$ and $\varepsilon_{2}$. Then, $\varepsilon_{1}=\Delta R / R, \varepsilon_{2}=\left(2 \pi R-2 \pi R^{\prime}\right) /(2 \pi R)=\Delta R / R$. This equation suggests the axial and tangential compressive strain will be equal if the droplet has a circular shape. Certainly, a deviation from a circular shape for the droplet is possible, which will affect the stress distribution in the surface layer.

Several mechanics models have been developed for buckling in heterogeneous structure of this type. ${ }^{4,5}$ For a prestretched thin film (prestrain, $\varepsilon$ ) of thickness $h_{f}$ and elastic modulus $E$ on a substrate of modulus $E_{s}$, releasing the prestrain leads to purely sinusoidal displacement distributions. Mechanics models give the wavelength $\lambda=\pi h_{f} / \sqrt{\varepsilon_{c r}}$ and amplitude $A=h_{f} \sqrt{\left(\varepsilon / \varepsilon_{c r}\right)-1}$,, 5 where $\varepsilon_{c r}=1 / 4\left(3 \bar{E}_{s} / \bar{E}\right)^{2 / 3}$ is the critical buckling strain given in terms of the plane-strain elastic moduli $\bar{E}$ and $\bar{E}_{s}$ of the film and substrate. The following relationship can be obtained:

$$
h_{f}=\sqrt{\frac{\lambda^{2} \varepsilon}{\pi^{2}}-A^{2}} .
$$

Both of the wavelength and amplitude can be informed from our AFM measurements. From the expression of wavelength and critical buckling strain, we can see the wavelength is proportional to $\left(\bar{E}_{s}\right)^{-1 / 3}$. Since the effective modulus of 30 nm particle-assembly is larger, the observed buckling wave- 


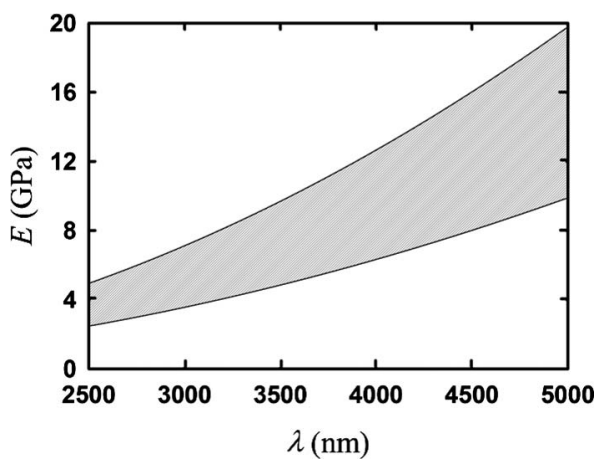

FIG. 3. Modulus of the surfactant monolayer as a function of wavelength. Wavelength and amplitude were measured by AFM. Due to different gap between the particle and the liquid surface and the variable arrangement of the particle-assembly within the liquid surface, the wavelengths are not constant and a range of wavelengths is shown in the $x$-axis.

length will be smaller. This is consistent with the AFM measurements shown in Figs. 2(b) and 2(c). During the transition phase shown in Fig. 1(a), the surface energy converts to strain energy. Before the buckling the strain energy stored in the surface film is

$$
W=\frac{1}{2} \int_{V} \varepsilon_{\alpha \beta} \sigma_{\alpha \beta} \mathrm{d} V=\frac{E \varepsilon^{2}}{1-\nu} \pi R^{2} h_{f},
$$

where $\sigma_{11}=\sigma_{22}=(E \varepsilon) /(1-\nu)$, and $\nu$ is the Poisson ratio of surface film. If we assume more than half of the change of surface free energy $\Delta G$ is converted to strain energy [Eq. (2)], i.e., $\Delta G / 2 \leq W \leq \Delta G$, then we can get

$$
\frac{\gamma}{\varepsilon h_{f}} \leq \frac{E}{1-\nu} \leq \frac{2 \gamma}{\varepsilon h_{f}}
$$

Substituting Eq. (3) into Eq. (1) yields

$$
\frac{\lambda^{2} \gamma}{\pi^{2} h_{f}\left(A^{2}+h_{f}^{2}\right)} \leq \frac{E}{1-\nu} \leq \frac{2 \lambda^{2} \gamma}{\pi^{2} h_{f}\left(A^{2}+h_{f}^{2}\right)} .
$$

Here, we treat the film as incompressible or in other words, $\nu=0.5$. In addition, due to the monolayer covering on the water surface, the surface tension $\gamma$ is $0.025 \mathrm{~N} / \mathrm{m}$.

While above analysis predicts constant amplitude $(A)$ and wavelength $(\lambda)$ throughout the buckling features, our measured amplitudes and wavelengths varied over the entire nanoparticles surfaces due to the nonuniform water gap between the upper surfactant layer and the underneath nanoparticles bank, generally a decreasing $A$ and an increasing $\lambda$ from the edge of the droplet to the center. We chose $80 \mathrm{~nm}$, which is the largest amplitude from the AFM measurements on nanoparticle surfaces, as the amplitude for the buckled surfactants. Because the surfactant molecule is of 12-carbon chain, we approximated the layer thickness $\left(h_{f}\right)$ as $2 \mathrm{~nm}$. Figure 3 shows calculated $E-\lambda$ relationship from Eq. (4). From this figure, the fluctuation in buckling wavelength delivered a broad distribution of elastic modulus for the surfactant layer. When less water exists between the surfactant layer and top of the nanoparticles, the substrate modulus $\left(E_{s}\right)$ in the expression of critical buckling strain will be higher, leading to a smaller buckling wavelength. In Fig. 3, the smallest modulus, $4 \mathrm{GPa}$, corresponded to the smallest wavelength, or in other words, a condition of no water between the surfactant layer and top of the nanoparticles. In this case, the modulus, i.e, $4 \mathrm{GPa}$, is the modulus of the surfactant layer, similar to the reported modulus of an ultrathin polystyrene (PS) film. ${ }^{7}$ Essentially, not only do surfactant and PS share similar atomic compositions but their similar molecular packing at the nanometer scale has downplayed their original difference in molecular weight to the observed mechanical properties. ${ }^{13}$ Moreover, this aforementioned buckling model is suitable for all two dimensional problems. For a herringbonetype buckling, Audoly and Boudaoud have had an asymptotic solution. ${ }^{14}$ From their solution, the kink angle of a herringbone pattern, which is $\theta$ in Fig. 2(a), should be determined by the ratio of axial and tangential compressive strains or $\tan (\theta / 2)=\varepsilon_{1} / \varepsilon_{2}$. Therefore, when the drying front is of a circle shape, the kink angle is $90^{\circ}$ as what we observed.

In summary, we discovered a buckling phenomenon occurred during the evaporation of an aqueous droplet containing surfactant molecules and nanoparticles. We further hypothesized a transient surface force at the triple interfaces of air-water-nanoparticles to be responsible for the observed 2D zig-zag patterns. Using solid mechanics theory, we analyzed the buckled features by suggesting an elastic modulus of $4 \mathrm{GPa}$ for the surfactant monolayer. We envision a deep understanding of the evaporation process before the cracking of a drying droplet is fundamentally important since it may shed light to crack growth mechanism and can lead to efforts eliminating or utilizing material failure. Moreover, this work offers an alternative means to measure mechanical properties of ultrathin films with great simplicity.

The authors gratefully acknowledge the partial financial support from the National Science Foundation (Project No. CMMI 0900644; Program Manager: Dr. Mary Toney) and the Agilent Technologies Foundation (Project No. 09US-672; Program Director: Dr. Jack Wenstrand). We also appreciate the reviewer for presenting us insightful comments.

${ }^{1}$ K.-H. Jeong, J. Kim, and L. P. Lee, Science 312, 557 (2006).

${ }^{2}$ D. Y. Khang, H. Jiang, Y. Huang, and J. A. Rogers, Science 311, 208 (2006).

${ }^{3}$ X. Chen and J. W. Hutchinson, ASME J. Appl. Mech. 71, 597 (2004).

${ }^{4}$ Z. Y. Huang, W. Hong, and Z. Suo, J. Mech. Phys. Solids 53, 2101 (2005).

${ }^{5}$ C. M. Stafford, C. Harrison, K. L. Beers, A. Karim, E. J. Amis, M. R. Vanlandingham, H. C. Kim, W. Volksen, R. D. Miller, and E. E. Simonyi, Nature Mater. 3, 545 (2004).

${ }^{6}$ C. M. Stafford, B. D. Vogt, C. Harrison, D. Julthongpiput, and R. Huang, Macromolecules 39, 5095 (2006).

${ }^{7}$ J. S. Huang, M. Juszkiewicz, W. H. de Jeu, E. Cerda, T. Emrick, N. Menon, and T. P. Russell, Science 317, 650 (2007).

${ }^{8}$ D. D. Robert, B. Olgica, F. D. Todd, H. Greb, R. N. Sidney, and A. W. Thomas, Nature (London) 389, 827 (1997).

${ }^{9}$ J. M. Guerra, M. Srinivasarao, and R. S. Stein, Science 262, 1395 (1993).

${ }^{10}$ A. F. Routh and W. B. Russel, Langmuir 15, 7762 (1999).

${ }^{11}$ S. T. Milner, J. F. Joanny, and P. Pincus, Europhys. Lett. 9, 495 (1989).

${ }^{12}$ D. M. Holmes, F. Tegeler, and W. J. Clegg, J. Eur. Ceram. Soc. 28, 1381 (2008).

${ }^{13}$ T. Oshiro, A. Backstrom, A. M. Cumberlidge, K. W. Hipps, U. Mazur, S. P. Pevovar, D. F. Bahr, and J. Smieja, J. Mater. Res. 19, 1461 (2004).

${ }^{14}$ B. Audoly and A. Boudaoud, J. Mech. Phys. Solids 56, 2444 (2008). 